\title{
La digitalizzazione dei papiri medici di Antinoupolis: caratteristiche e particolarità
}

\author{
Francesca Corazza
}

Il progetto CPGM - Corpus dei Papiri Greci di Medicina Online ha permesso la digitalizzazione di c. 280 papiri greci di contenuto medico - un numero progressivamente in crescita - e il loro inserimento sulla piattaforma Papyri.info poi confluita nella banca dati del Digital Corpus of Literary Papyrology (DCLP) ${ }^{1}$. L'archivio digitale dei papiri medici include la maggior parte dei manoscritti provenienti da Antinoupolis ${ }^{2}, 27$ testi perlopiù in forma di codice che racchiudono un'ampia gamma di generi e contenuti, dai trattati teoretici ai ricettari, dai manuali anonimi alle opere della tradizione ippocratica, dai testi di medicina tradizionale a quelli iatromagici (Tab. 1). Una tale varietà di testi permette di presentare non soltanto le caratteristiche principali dell'edizione digitale, ma anche alcune particolarità che sono proprie dei testi semi-letterari e che più difficilmente si ritrovano nei papiri documentari. Attraverso alcuni esempi tratti dalle edizioni digitali dei papiri di Antinoupolis, il presente contributo intende illustrare le modalità di conversione del testo dal formato cartaceo a quello elettronico e alcune difficoltà che si sono incontrate.

L'edizione digitale è costituita da sei componenti fondamentali, ovvero: la scheda dei metadati, il front matter, il testo annotato, il line-by-line commentary e, laddove presente, la traduzione in una o più lingue moderne.

La scheda dei metadati consente di visualizzare in maniera sintetica le informazioni principali utili all'identificazione del papiro come la datazione, il luogo di provenienza, il genere, l'autore e l'editore. Questi dati, derivati automaticamente dai

${ }^{1}$ La digitalizzazione dei papiri di Antinoupolis, così come il presente contributo, fa parte del progetto "Online Humanities Scholarship: A Digital Medical Library Based on Ancient Texts" (ERGAdG-2013-DIGMEDTEXT, Grant Agreement No. 339828, Principal Investigator Prof. Isabella Andorlini), condotto su fondi dello European Research Council presso l'Università di Parma ( $<$ http://www.papirologia.unipr.it/ERC $>$ ). Sui progetti digitali di papirologia e risorse online si veda anche Andorlini/Reggiani, Edizione; Bagnall, Integrating; Delattre/Heilporn, Resources; Reggiani, Digital Papyrology I, passim; Id., Tavolette; Id., Project; Id., Perspectives.

${ }^{2}$ Ad oggi, PSI Ant. inv. NN 16-12-2013 (LDAB 642454) e PSI Ant. inv. 320 A (LDAB 642455), editi nel 2015 da Del Corso/Pintaudi, Papiri, nonché P.Ant. III 146 (LDAB 6327) e 148 (LDAB 5996), di contenuto incerto, non sono stati ancora digitalizzati. 
cataloghi Trismegistos e Leuven Database of Ancient Books, rendono possibile la ricerca di un testo o di un contenuto specifico tramite l'uso di parole chiave e contribuiscono a rendere facilmente accessibile una collezione così estesa.

Il front matter precede invece il testo fornendo una descrizione completa, seppur concisa, del papiro. A differenza della scheda dei metadati, le informazioni sono riportate in maniera più estesa e sono approfondite soprattutto in merito alle caratteristiche materiali e testuali più rilevanti, al contenuto, alle caratteristiche paleografiche e datazione del manoscritto.

Il testo annotato, da cui si ricava l'apparato critico posto in calce, rappresenta il cuore dell'edizione digitale. L'edizione critica più recente costituisce generalmente la base del testo, ma si fa costante riferimento alle edizioni precedenti in caso di proposte editoriali alternative. Codificato in linguaggio Leiden+, il testo annotato permette di veicolare attraverso i tag informazioni di diverso tipo automaticamente convertite in codice XML secondo lo standard $\mathrm{TEI} /$ EpiDoc e visualizzate secondo convenzioni predefinite ${ }^{3}$.

Essendosi convenuto di dedicare precisa attenzione anche alla storia editoriale dei testi, nella digitalizzazione di papiri medici è piuttosto frequente il ricorso al tag |alt| per indicare le letture alternative dell'editore di riferimento, e al tag |ed| per indicare le letture di altri editori. Le differenze tra le letture possono interessare l'interpretazione di una singola lettera o di un'intera parola, e per facilitare confronto tra le diverse edizioni, soprattutto in caso di testi particolarmente controversi, riediti e commentati da numerosi studiosi, si può aggiungere al tag |ed| (che di default stabilisce un confronto tra l'edizione di riferimento e l'edizione immediatamente precedente) l'indicazione =ed.pr. o =nome dell'editore. Si veda ad esempio P.Ant. I $28(b)(<h t t p: / / l i t p a p . i n f o / d c l p / 60189>)^{4}$, un unico frammento di un codice di pergamena datato al tardo III-inizi del IV sec. d. C.:

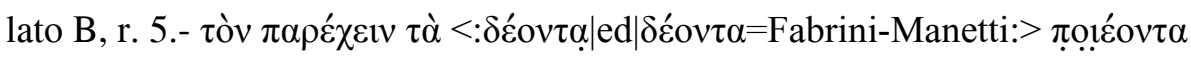

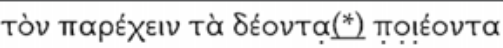

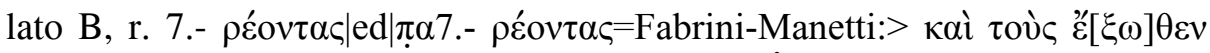
$<: *$ slanting-stroke*|ed|vac.? .=Fabrini-Manetti: $>\dot{\varepsilon}[v] \tau \mid \underline{ि} \sigma \mathrm{l}$

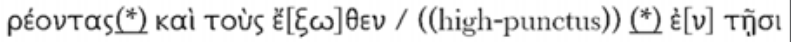

Il nome dell'editore è riportato in apparato come segue:

$\wedge$ B.5. Séovta Fabrini-Manetti

\footnotetext{
${ }^{3}$ Per le specifiche linee guida del linguaggio Leiden+, cf.<http://papyri.info/docs/leiden_plus >

${ }^{4}$ Edd.: Colin H. Roberts, P.Ant. I 28; Pierangelo Fabrini / Daniela Manetti, CPF I.2* [18] 1.
} 
B.7. vac. ? . Fabrini-Manetti

All'interno di ogni tag è possibile annidarne un altro, come avviene nel caso di una regolarizzazione formale contenuta all'interno di una lettura alternativa. Le varianti fonetiche di scambio di vocali e/o di consonanti costituiscono il tipo di regolarizzazione più comune nei papiri medici (così come solitamente in tutti gli altri testi su papiro). Ecco come viene visualizzata, rispettivamente in linguaggio Leiden+, a testo e in apparato, una stringa di tag annidati in P.Ant. III 183 (<http://litpap.info/dclp/60191>) $)^{5}$, un codice papiraceo databile al VI sec. d.C. sopravvissuto in 4 frammenti piuttosto lacunosi e contenente parte degli Aforismi di Ippocrate (Aph. III 20-29, $31+$ IV 1, 4-5):

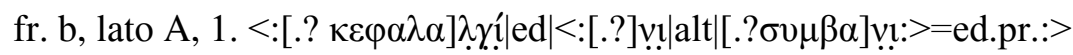

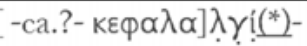

A.b.Ar. [-ca.?- ]

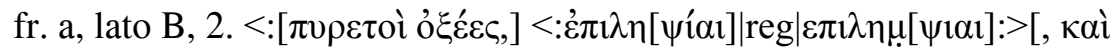
$\left.\tau{ }^{\prime \prime} \lambda \lambda \alpha\right]|\operatorname{ed}|[. ?] \varepsilon \pi \imath \lambda \eta \mu[\psi 1 \alpha 1 . ?]=$ ed.pr.: $>$

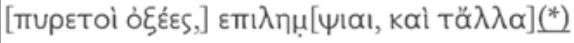

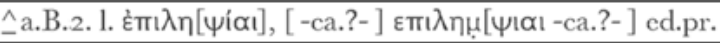

Come si può notare, i tag risultano efficaci e chiari nella loro visualizzazione. Tuttavia, in alcuni casi riprodurre esattamente in linguaggio Leiden+ le informazioni contenute nell'edizione cartacea può presentare alcune difficoltà . La regolarizzazione, ad esempio, viene sempre visualizzata in apparato nel rispetto del testo originale anche quando nell'edizione di riferimento è riportata all'interno del testo principale ${ }^{7}$, come si può vedere in P.Ant. I 28:

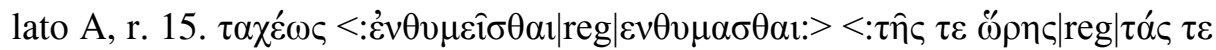

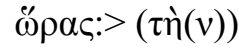

\footnotetext{
${ }^{5}$ Edd.: John W. B. Barns / Henrik Zilliacus, P.Ant. III 183; Isabella Andorlini, CPF I.2* [18] 4.

${ }^{6}$ Sulla necessità di implementare la piattaforma SoSOL con categorie informative specifiche per i testi medici e su alcune proposte di integrazione dei dati esistenti, cf. Reggiani, Project, 343 ss.; Id., Perspectives, passim; Id., Concept, passim.

${ }^{7}$ Sulle problematiche legate alla distinzione tra varianti filologiche e grafiche 'principali' e 'secondarie', e sulle possibilità offerte dall'edizione digitale di superare tali distinzioni in favore di una concezione più fluida di trasmissione del testo, cf. Reggiani, Digital Papyrology I, 265 ss.
} 


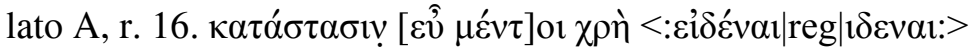

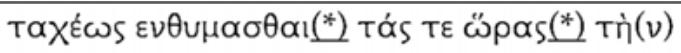

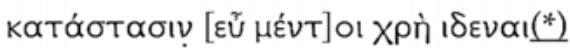

L'apparato è visualizzato come segue:

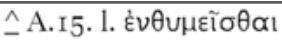

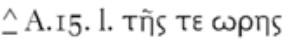

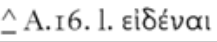

Questo tipo di visualizzazione viene generata in automatico secondo le regole del codice XML che attualmente non consentono di modificare il testo digitale in tal senso in conformità con l'edizione cartacea.

Tra le caratteristiche più frequentemente segnalate nel testo annotato ricordiamo anche le abbreviazioni realizzate dallo scriba tramite segni convenzionali. Oltre alle abbreviazioni comunemente utilizzate per le unità di misura, in P.Ant. III 127 (<http://litpap.info/dclp/65340>) $)^{8}$ sono presenti numerosi casi di sopralineatura di $v$ con tratto orizzontale, la sinusoide (s) è utilizzata come abbreviazione di $\alpha$, e il segno .//. sostituisce l'intera parola $\varepsilon ı \sigma$. Le diverse tipologie di abbreviazione sono segnalate nel testo digitale senza distinzione tramite l'uso standard delle parentesi tonde':

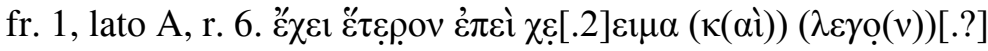

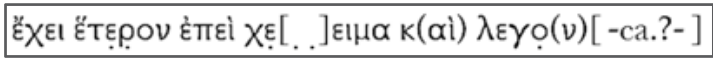

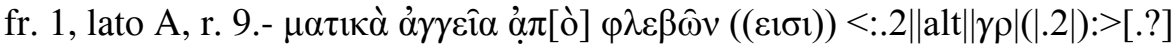

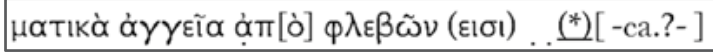

Ciò nonostante, l'edizione digitale cerca di riprodurre quanto più fedelmente possibile il layout dei papiri preservandone anche a livello visivo la struttura del testo. L'utilizzo di appositi marcatori permette, ad esempio, di segnalare la posizione rientrata o sporgente di un rigo rispetto al corpo del testo (eisthesis / ekthesis $)^{10}$, come avviene in P.Ant. I 28, contenente l'incipit di Aforismi I introdotto dal titolo dell'opera e dal nome dell'autore:

\footnotetext{
${ }^{8}$ Edd.: John W. B. Barns / Henrik Zilliacus, P.Ant. III 127; Andorlini, Tradizione, 12-27.

${ }^{9}$ Cf. Reggiani, Concept, $\S 4.6$.

${ }^{10} \mathrm{La}$ marcatura (numero del rigo, indent) / (numero del rigo, outdent) sostituisce la marcatura
} 


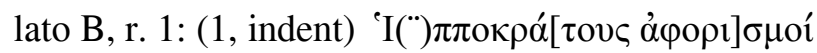

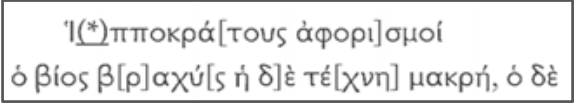

e in P.Ant. III 183, dove il numerale $\Delta(=4)$ seguito da $\alpha(=1)$ in posizione rientrante rispetto ai righi seguenti segnalava verosimilmente l'inizio del primo aforisma del quarto libro ${ }^{11}$ :

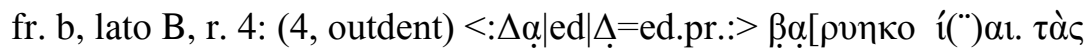

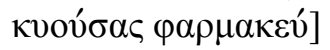

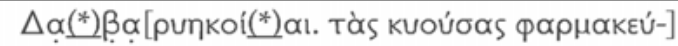

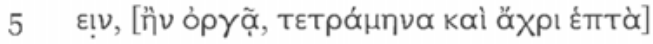

I papiri medici di Antinoupolis si rivelano particolarmente interessanti sotto il profilo della struttura del testo perché alcuni codici contengono scolii e annotazioni lungo il margine laterale e inferiore. Per indicare l'aggiunta di annotazioni marginali si utilizza la marcatura $1, \mathrm{~ms} / \mathrm{md} / \mathrm{minf} / \mathrm{msup}$ rispettivamente per il margine sinistro, destro, inferiore e superiore, dove il numerale corrisponde al primo rigo del testo marginale. È possibile indicare se gli scolii sono stati aggiunti da una seconda o eventualmente terza mano utilizzando la marcatura (hand2), (hand3).

Nonostante le aggiunte appaiano chiaramente separate rispetto al testo principale, non è possibile riprodurre con esattezza la loro posizione né il loro reciproco rapporto contenutistico e semantico ${ }^{12}$. La corrispondenza tra i righi del testo principale e quelli del testo in margine, scritto normalmente in caratteri più piccoli, è soltanto indicativa poiché il testo secondario viene convenzionalmente interposto e non affiancato a quello principale. Inoltre, a livello visivo non vi è distinzione tra le annotazioni lungo il margine sinistro e quello destro, o tra il margine superiore e quello inferiore, come si vede nell'esempio riportato (P.Ant. III $183(a))$ :

\footnotetext{
*eisthesis* / *ekthesis* precedentemente utilizzata.

${ }^{11}$ Cf. CPF I.2* [18] 4.

12 Un altro esempio di visualizzazione imperfetta del testo correttamente espresso in linguaggio Leiden+ e XML è riportata da Bertonazzi, P.Strasb.
} 


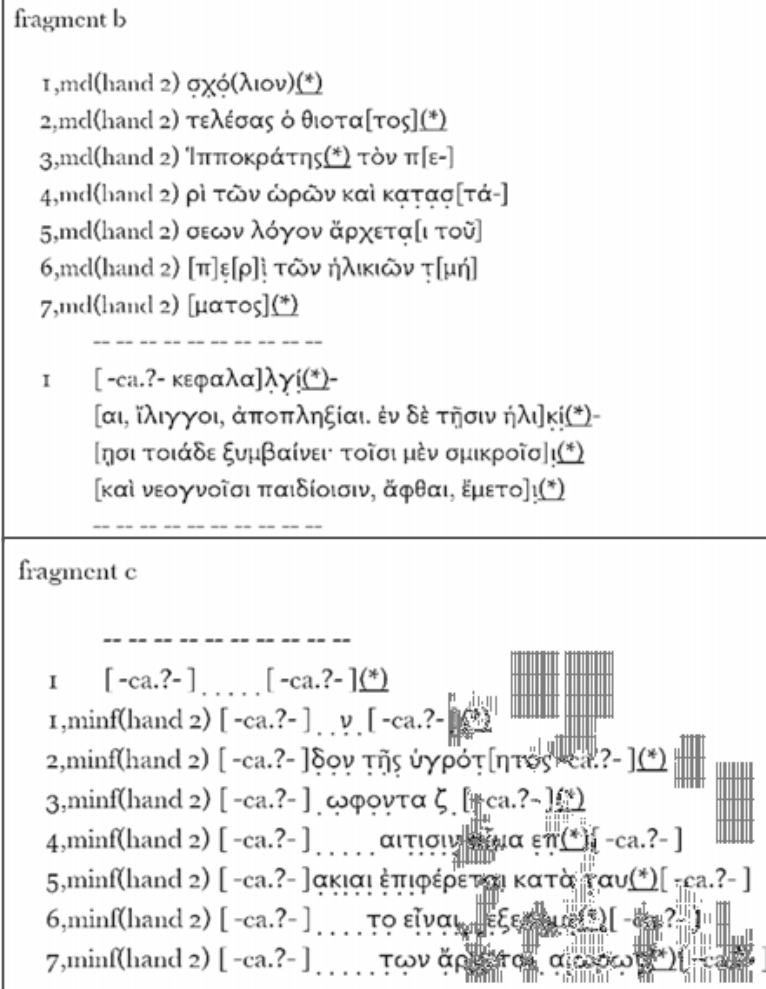

Diverso è il caso delle aggiunte marginali di parole e segni diacritici segnalate tramite i tag \|left:|| oppure ||right:\|. Si veda e.g. P.Ant. III 186 (SoSOL 2016 253) ${ }^{13}$ :

lato B, fr. 14 r.8: (8, eisthesis) $\|$ left: $<: .2 \mid$ alt|*diple*:> $\mid \|$ $\rho$ ó $\pi[o v . ~ * d i p l e-$ obelismene* *diple-obelismene* ${ }^{*} \alpha \lambda$ o. *diple-obelismene* ]

L'utilizzo di due diverse tipologie di marcatura per le aggiunte in margine permette di distinguere la loro diversa natura. Nel primo caso, la numerazione dei righi associata alla marcatura $\mathrm{ms} / \mathrm{md} / \mathrm{msup} / \mathrm{minf}$ indica l'aggiunta di un testo che, pur mostrando attinenza con quello principale, conserva una propria autonomia. Nel secondo caso, invece, i tag \|left:|| / |right:|| segnalano l'apposi-zione di parole o segni che mantengono una continuità con il testo principale, come avviene per l'aggiunta di omissioni o di segni diacritici.

\footnotetext{
${ }^{13}$ Edd.: John W. B. Barns / Henrik Zilliacus, P.Ant. III 186; Daniela Manetti, CPF I.2* [14] 2.
} 
Sotto il profilo della disposizione formale del testo, i papiri medico-magici presentano non di rado caratteristiche non convenzionali riproducibili nell'edizione digitale tramite specifici accorgimenti. Un esempio è dato da P.Ant. II 66 (<http://www.litpap.info/dclp/64757>) $)^{14}$, un codice papiraceo del V sec. d.C. contenente una raccolta di ricette iatromagiche. La formula ai righi 40-43 è inscritta in un rettangolo, ed è trascritta in linguaggio Leiden+ utilizzando la marcatura \#\#\#:

40. [.?]\#\#\#1w1

41. [.?]\#\#\# $\alpha \omega \omega$

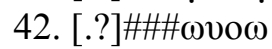

43. [.? $\# \# \# .100$

Il testo viene visualizzato come segue:

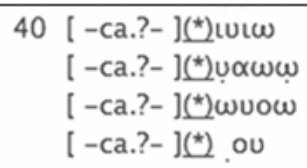

Per quanto l'edizione digitale, a differenza di quella cartacea, non riproduca visivamente il particolare layout del testo originale, è possibile recuperare questa informazione facendo riferimento all'apparato critico, che indica infatti:

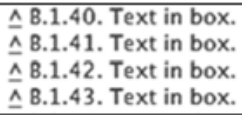

In casi più complessi, invece, non è possibile veicolare informazioni più specifiche tramite l'apparato ma ci si deve affidare unicamente al line-by-line commentary, che fornisce informazioni supplementari in merito alle caratteristiche del testo.

Il commento ai righi contiene spesso riferimenti al rapporto tra i papiri e la tradizione indiretta. Sei codici medici da Antinoupolis conservano porzioni dei trattati di Ippocrate o Galeno ${ }^{15} \mathrm{e}$, trattandosi di testi attribuiti ad autori noti, è stato possibile confrontare la versione papiracea con quella trasmessa dai manoscritti medievali. L'inserimento di riferimenti alle varianti testuali all'interno del commento anziché del testo annotato consente non solo di rendere l'apparato critico più agile e semplice da consultare, ma anche di mantenere distinti i diversi tipi di informazione. Si è scelto di segnalare principalmente i punti di continuità e di rottura con la tradizione medievale al fine di integrare il testo lacunoso del

\footnotetext{
${ }^{14}$ Barns/Zilliacus, P.Ant. II 66, 47-9; Betz, Papyri, 304-5; Suppl.Mag. II 94, p. 214; Corazza, Medicina.

${ }^{15}$ P.Ant. I 28; III 186 + 139; III 183; III 184; II 86; III 185.
} 
papiro o evidenziare possibili tradizioni indipendenti.

Infine, la traduzione del testo greco in una o più lingue moderne, laddove presente nelle edizioni di riferimento, completa l'edizione digitale. Le molteplici traduzioni di uno stesso testo - anche in una stessa lingua - consentono di mettere a confronto le interpretazioni di passi controversi, come avviene in P.Ant. II 66 ai rr. 45-60, in cui i sintomi elencati sono stati attribuiti da editori diversi ad una ferita o al mal di testa ${ }^{16}$.

L'edizione digitale mette insieme in modo rigoroso e dinamico i risultati degli studi fatti sul papiro a diversi livelli - linguistico, filologico, e testuale. Essa si presenta come una riproduzione completa della versione cartacea corrispondente, destinata tuttavia a superare il modello canonico di indagine critica per diventare oggetto di una nuova indagine digitale, intertestuale e multi-modale ${ }^{17}$. Alla luce dei progressi compiuti fino ad ora, si auspica che i prossimi miglioramenti del sistema di marcatura e delle istruzioni di trasformazionee XSLT permettano di perfezionare la codifica e la visualizzazione dei dati contenuti nel testo annotato e di veicolare sempre maggiori informazioni non immediatamente accessibili attraverso le edizioni tradizionali.

\begin{tabular}{|l|c|c|c|c|}
\hline & TM & Datazione & Genere & \multicolumn{1}{c|}{ DCLP } \\
\hline P.Ant. III 138 & 63874 & $100-299$ & trattato & http://litpap.info/dclp/63874 \\
\hline P.Ant. III 129 & 64139 & $200-299$ & trattato & http://litpap.info/dclp/64139 \\
\hline P.Ant. III 133 & 64330 & $200-399$ & frammento medico & http://litpap.info/dclp/64330 \\
\hline P.Ant. I 28 & 60189 & $400-499$ & Hp. Aph. & http://litpap.info/dclp/60189 \\
\hline P.Ant. III 134 & 64482 & $300-399$ & Ricette & http://litpap.info/dclp/64482 \\
\hline $\begin{array}{l}\text { P.Johnson + } \\
\text { P.Ant. III 214 }\end{array}$ & 64598 & $350-450$ & erbario illustrato & http://litpap.info/dclp/64598 \\
\hline $\begin{array}{l}\text { P.Ant. III 186 } \\
+\end{array}$ & 59961 & $500-599$ & Gal. Comp.med.gen. & http://litpap.info/dclp/59961 \\
$\begin{array}{l}\text { P.Ant. III 139 } \\
\text { P.Ant. II 66 }\end{array}$ & 64757 & $400-499$ & ricette iatromagiche & http://litpap.info/dclp/64757 \\
\hline P.Ant. III 140 & 64896 & $400-599$ & ricette iatromagiche & http://litpap.info/dclp/64896 \\
\hline P.Ant. III 137 & 64900 & $400-599$ & frammento medico & http://litpap.info/dclp/64900 \\
\hline
\end{tabular}

${ }^{16}$ Nella traduzione di Robert W. Daniel / Franco Maltomini, Suppl.Mag. II 94, p. 214, la formula è riferita ad una ferita causata verosimilmente dal morso di un animale velenoso: "(voces magicae) I say to you, wound, do not throb, do not become inflamed, do not give rise to pains, do not cause humor, do not cause blackening, do not give rise to gangrene. For if you throb or become inflamed or give rise to pains or cause humor o cause blackening, or give rise to gangrene, ...". Il testo tradotto da Roy D. Kotansky ap. Betz, Papyri, 305 fa invece riferimento ad un rimedio contro il mal di testa: "to you I speak, pounding headache: don't throb, don't rage, don't shake the teeth, don't produce mucus, don't produce a 'black-out', don't stir up convulsions. For if there is throbbing, raging, grinding of teeth, production of mucus, producing of a 'black-out' or stirring of a convulsion ...".

17 Sul concetto di "meta-papyrus" e sulle nuove frontiere della papirologia digitale si veda soprattutto Reggiani, Digital Papyrology I, 268 ss. 


\begin{tabular}{|c|c|c|c|c|}
\hline P.Ant. III 183 & 60191 & $500-599$ & Hp. Aph. & http://litpap.info/dclp/60191 \\
\hline P.Ant. III 184 & 60192 & $575-625$ & Hp. Mul., Superfoet. & http://litpap.info/dclp/60192 \\
\hline P.Ant. II 86 & 60193 & $500-599$ & Hp. Aph. & http://litpap.info/dclp/60193 \\
\hline P.Ant. III 185 & 60190 & $500-599$ & $\begin{array}{l}\text { Hp. Aph., Nat.hom., } \\
\text { Sal.vict.rat. }\end{array}$ & http://litpap.info/dclp/60190 \\
\hline P.Ant. III 123 & 65077 & $500-599$ & materia medica & http://litpap.info/dclp/65077 \\
\hline P.Ant. III 124 & 65075 & $500-599$ & trattato & http://litpap.info/dclp/65075 \\
\hline P.Ant. III 125 & 65076 & $500-599$ & trattato & http://litpap.info/dclp/65076 \\
\hline P.Ant. III 128 & 65074 & $500-599$ & trattato & http://litpap.info/dclp/65074 \\
\hline P.Ant. III 130 & 65078 & $500-599$ & ricette & http://litpap.info/dclp/65078 \\
\hline P.Ant. III 131 & 65072 & $500-599$ & frammento medico & http://litpap.info/dclp/65072 \\
\hline P.Ant. III 132 & 65079 & $500-599$ & ricette & http://litpap.info/dclp/65079 \\
\hline P.Ant. III 135 & 65080 & $500-599$ & ricette & http://litpap.info/dclp/65080 \\
\hline P.Ant. III 136 & 65073 & $500-599$ & frammento medico & http://litpap.info/dclp/65073 \\
\hline P.Ant. II 64 & 65082 & $500-599$ & ricette & http://litpap.info/dclp/65082 \\
\hline P.Ant. III 126 & 65233 & $500-699$ & enciclopedia medica & http://litpap.info/dclp/65233 \\
\hline P.Ant. III 127 & 65340 & $600-699$ & trattato; ricette & http://litpap.info/dclp/65340 \\
\hline
\end{tabular}

\section{Bibliografia}

Andorlini, Tradizione = Isabella Andorlini, I papiri e la tradizione medievale nella ricettazione dei testi medici tardoantichi, in: A. Garzya (ed.), Tradizione e ecdotica dei testi medici tardoan-tichi e bizantini, Napoli 1992, 13-27.

Andorlini/Reggiani, Edizione = Isabella Andorlini / Nicola Reggiani, Edizione e ricostruzione digitale dei testi papiracei, in: N. Palazzolo (ed.), Diritto romano e scienze antichistiche nell'era digitale (Collectanea Graeco-Romana 10), Torino 2012, 131-46.

Bagnall, Integrating = Roger S. Bagnall, Integrating Digital Papyrology, in J. McGann (ed.), Online Humanities Scholarship. The Shape of Things to Come, Houston 2012, URL: <https://archive.nyu. edu/handle/2451/29592 >.

Baumann, Son = Ryan Baumann, The Son of Suda On-Line, in S. Dunn / S. Mahony (edd.), The Digital Classicist, Bulletin of the Institute of Classical Studies Supp. 122, London 2013, URL: $<$ http://ryanfb.github.io/papers-BICS/sosol-bics-draft.pdf>.

Bertonazzi, P.Strasb. = Francesca Bertonazzi, Digital Edition of P.Strasb. inv. 1187: Between the Papyrus and the Indirect Tradition, in Proceedings of 28th Congress of Papyrology (1-6 august 2016, Barcelona), Barcelona 2018, in corso di pubblicazione.

Betz, Papyri = Hans D. Betz, The Greek Magical Papyri in Translation, Chicago 1986.

Corazza, Medicina $=$ Francesca Corazza, Il rapporto tra medicina templare e tradizionale nella testimonianza dei papiri greci di Antinoupolis, in: N. Reggiani / F. Bertonazzi (edd.), Parlare la medicina: fra lingue e culture, nello spazio e nel tempo. Atti del Convegno Internazionale (Parma, 5-7 Settembre 2016), Firenze 2018, 80-8.

Del Corso/Pintaudi, Papiri = Lucio Del Corso / Rosario Pintaudi, Papiri letterari dal Museo Egizio del Cairo e una copertina di codice da Antinoupolis, in: L. Del Corso / F. De Vivo / A. Stramaglia (edd.), Nel segno del testo. Edizioni, materiali e studi per Oronzo Pecere, Firenze 2015, 3-29.

Delattre/Heilporn, Resources = Alain Delattre / Paul Heilporn, Electronic Resources for GraecoRoman and Christian Egypt: A Review of the State of the Net, "Bibliotheca Orientalis" 71 (2014), 308-31. 
Reggiani, Concept $=$ Nicola Regggiani, The Digital Corpus of the Greek Medical Papyri and a New Concept of Digital Critical Edition, in: N. Reggiani (ed.), Digital Papyrology II. Case Studies on the Digital Edition of Ancient Greek Papyri, Berlin/Boston 2018, 3-61.

Reggiani, Digital Papyrology I = Nicola Reggiani, Digital Papyrology I, Berlin/Boston 2017.

Reggiani, Perspectives = Nicola Reggiani, The Corpus of Greek Medical Papyri and Digital Papyrology: New Perspectives from an Ongoing Project, in: M. Berti / F. Naether (edd.), Altertumswissenschaften in a Digital Age: Egyptology, Papyrology and Beyond. Proceedings of a Conference and Workshop in Leipzig, November 4-6, 2015, Leipzig 2016, URL: <http://nbnresolving.de/urn:nbn:de:bsz:15-qucosa-201726>.

Reggiani, Project = Nicola Reggiani, A Corpus of Literary Papyri Online: The Pilot Project of the Medical Texts via SoSOL, in: R. Lafer / K. Strobel (edd.), Antike Lebenswelten. Althistorische und papyrologische Studien, Berlin/New York 2015, 341-52.

Reggiani, Tavolette = Nicola Reggiani, Le tavolette della mente. Risorse digitali e Antichistica: il caso della Papirologia, in: A. M. Tammaro (ed.), Umanisti e risorse digitali, Parma 2012, 88-110. 